\title{
An improved substructural identification strategy for large structural systems
}

\author{
Thanh N. Trinh ${ }^{1}$ and C. G. Koh ${ }^{2}$ \\ ${ }^{1}$ Applied Computing and Mechanics Laboratory, Structural Engineering Institute, Station 18, \\ Swiss Federal Institute of Technology in Lausanne (EPFL), CH-1015 Lausanne, Switzerland \\ ngocthanh.trinh@epfl.ch, tel: +41216936372, fax: +41216934748 \\ ${ }^{2}$ Department of Civil Engineering, National University of Singapore, Singapore 117576 \\ cgkoh@nus.edu.sg
}

\begin{abstract}
To identify physical parameters of a large structural system, the computational challenges in dealing with a large number of unknowns are formidable. A divide-and-conquer approach is often required to partition the structural system into many substructures, each with much lesser unknowns for more accurate and efficient identification. Furthermore, in view of the ill-conditioned nature of inverse analysis, it is highly beneficial to adopt non-gradient based search methods such as genetic algorithm (GA). To this end, this paper presents a GA-based substructural identification strategy for large structural systems. As compared to some recent work on substructural identification, the proposed strategy presents two significant improvements: (a) the use of acceleration measurements to directly account for interaction between substructures without approximation of interface force, and (b) the use of an improved identification method based on multi-feature GA. In numerical simulations, the mass, damping and stiffness parameters of a 100-storey shear building, involving 202 unknowns, are identified with very good accuracy (mean error of less than 3\%) based on incomplete acceleration measurements with $10 \%$ noise. In addition, an experimental study on a 10-storey small-scale steel frame further validates the superior performance of the proposed strategy over complete structural identification.
\end{abstract}


KEY WORDS: system identification; divide-and-conquer; substructure; genetic algorithm; structural dynamics; experiment.

\section{INTRODUCTION}

Identification of physical parameters, such as mass, stiffness and/or damping, of a structure based on input and output (I/O) measurements is known as structural identification. Structural identification can be applied to calibrate the structural model and can also be used for the purpose of structural health monitoring (SHM) via tracking change in pertinent parameters [1]. With rapid advances in sensors, wireless communication and information technologies as well as awareness of SHM's long-term benefits by the authorities and users [2-3], structural identification has received considerable attention in recent years for three main functions: (a) to improve understanding and design, (b) to reduce inspection and maintenance costs and (c) to monitor the response severity and assess damage should the need arise, say after a natural disaster.

While many structural identification methods in time domain and frequency domain have been proposed [4-12], most of these methods have been tested only on structures of limited unknowns. For large structural systems, modelling often involves a large number of degrees of freedom (DOFs) and also likely a large number of unknown parameters in the identification procedure. Hence, if the complete structure is identified at one go, known as complete structural identification (CSI), the identification faces with three challenges: (a) difficulty in achieving convergence, (b) the need for a large number of sensors and (c) expensive computation for processing huge amount of data. To address these challenges, substructuring provides a good solution by dividing a large structure into many smaller and more manageable parts called substructures, for which identification can be carried out independently and more easily due to the reduced system size. This procedure is referred to as substructural identification (Sub-SI). 
There are five advantages of substructural identification. (1) Since dividing a large structure into many substructures allows for reducing the number of unknowns to be identified at one time, the likelihood of converging to a solution is improved. (2) It is not necessary to monitor the whole structure simultaneously. Instead, only the critical parts, where damage is likely to occur, need to be monitored, resulting in a significant reduction in the number of sensors required and more efficient management of data. (3) Modelling errors induced due to mathematical modelling can be reduced by using substructures not involving any uncertain boundary condition [13-14]. (4) The method can be used as "output-only" identification if the excitation force is outside the substructure of interest. (5) Since each substructure is independently identified, the identification of many substructures can be concurrently conducted using parallel computing. In particular, for SHM applications using smart sensing technology [15], Sub-SI can be employed as a distributed computing strategy in a hierarchical SHM system. Communication and data processing in this regard mainly take place in the group of local sensors, thereby reducing transmission of large amounts of data.

Koh et al. [16] were the first to formulate a Sub-SI method to identify structural parameters, using the extended Kalman filter (EKF) with a weighed global iteration algorithm. Yun and Lee [17] presented a Sub-SI method using an autoregressive and moving average with stochastic input model (ARMAX) [18] and a sequential prediction error method [19]. Although these Sub-SI methods are able to identify the structural parameters of structures, they require measurements of acceleration, velocity and displacement at all interface DOFs. Tee et al. [20] proposed two Sub-SI methods based on the classical methods of observer/Kalman filter identification (OKID) [21] and eigensystem realization algorithm [22]. A fairly large structural system of 50 DOFs was numerically studied under noise 5\%. These Sub-SI methods achieved good accuracy but required the measurement of accelerations at all DOFs (i.e. complete measurement). To avoid the need for complete measurement, Tee 
et al. further improved Sub-SI by integrating a condensed model identification and recovery method [23-24], with 5\% maximum noise level considered. The method needs at least one internal force applied within each substructure and is thus limited in its application.

Substructural identification using the artificial neural network as the "search engine" was first presented by Yun and Bahng [25]. The results showed that this method could identify structural parameters with incomplete measurements of mode shapes and natural frequencies. Xu [26] recently proposed a neural-network Sub-SI method to identify stiffness and damping values of a shear building of 50 DOFs using acceleration measurements.

The previous mentioned Sub-SI methods identify stiffness and damping parameters but assume known mass parameters. In contrast, the problem of identifying unknown stiffness, damping and mass parameters simultaneously is much more difficult. To address this problem, Koh et al [27] proposed a Sub-SI method that was able to identify simultaneously stiffness, damping and mass parameters using genetic algorithm (GA). An advantage of the method is that only acceleration measurements are used to account for the interaction effect by employing the concept of "quasi-static" displacement vector. The method decomposes displacements of internal DOFs into quasi-static displacement and "relative" displacement. The equations of motion of a substructure were derived in terms of relative displacements through the use of the influence coefficient matrix. An assumption to allow for solving these equations was necessary, i.e. all the velocity-dependent components in the interface forces are to be negligible. Nevertheless, inaccuracy could result from (a) using relative displacement (since it involves computation of an influence coefficient matrix) rather than using directly acceleration measurements, and (b) neglecting the velocity-dependent components. In addition, since the GA adopted in this Sub-SI method conducts search on only one population, it is difficult to have a good trade-off between the exploration of new solutions and the exploitation of the refined solutions during search progress. Furthermore, although several 
Sub-SI methods have recently been developed [28-30], none of these Sub-SI methods has been tested experimentally.

This paper presents an improved substructural identification strategy for large structural systems and demonstrates its performance in both numerical an experimental studies. The improvement of identification accuracy is derived from two aspects. (a) This strategy employs directly acceleration measurements to account for interaction effects at the interface DOFs (avoiding the use of relative acceleration and the assumption of neglecting interface velocitydependent force). (b) Furthermore, this strategy utilizes a multi-feature GA algorithm to enhance its search capability for each substructure.

\section{SUBSTRUCTURAL FORMULATION}

To illustrate the concept of substructuring, consider a shear building as shown in Figure 1(a) which is represented by a lumped mass system as shown in Figure 1(b). Considering a substructure with DOFs denoted by vector $\mathbf{u}_{\mathrm{j}}$ as shown in Figure 1(c), the equations of motion for the full structure can be written in the following partition form [27]

$$
\left[\begin{array}{ccc}
\mathbf{M}_{r r} & \mathbf{M}_{r j} & \mathbf{0} \\
\mathbf{M}_{j r} & \mathbf{M}_{j j} & \mathbf{M}_{j i} \\
\mathbf{0} & \mathbf{M}_{i j} & \mathbf{M}_{i i}
\end{array}\right]\left\{\begin{array}{l}
\ddot{\mathbf{u}}_{r} \\
\ddot{\mathbf{u}}_{j} \\
\ddot{\mathbf{u}}_{i}
\end{array}\right\}+\left[\begin{array}{ccc}
\mathbf{C}_{r r} & \mathbf{C}_{r j} & \mathbf{0} \\
\mathbf{C}_{j r} & \mathbf{C}_{j j} & \mathbf{C}_{j i} \\
\mathbf{0} & \mathbf{C}_{i j} & \mathbf{C}_{i i}
\end{array}\right]\left\{\begin{array}{l}
\dot{\mathbf{u}}_{r} \\
\dot{\mathbf{u}}_{j} \\
\dot{\mathbf{u}}_{i}
\end{array}\right\}+\left[\begin{array}{ccc}
\mathbf{K}_{r r} & \mathbf{K}_{r j} & \mathbf{0} \\
\mathbf{K}_{j r} & \mathbf{K}_{j j} & \mathbf{K}_{j i} \\
\mathbf{0} & \mathbf{K}_{i j} & \mathbf{K}_{i i}
\end{array}\right]\left\{\begin{array}{l}
\mathbf{u}_{r} \\
\mathbf{u}_{j} \\
\mathbf{u}_{i}
\end{array}\right\}=\left\{\begin{array}{l}
\mathbf{P}_{r} \\
\mathbf{P}_{j} \\
\mathbf{P}_{i}
\end{array}\right\}
$$

where $\mathbf{M}, \mathbf{C}, \mathbf{K}$ are the mass, damping, and stiffness matrices, respectively, $\mathbf{u}, \dot{\mathbf{u}}, \ddot{\mathbf{u}}$ are the displacement, velocity and acceleration vectors, respectively, and $\mathbf{P}$ is the input force vector, subscript $j$ denotes interface DOFs of the substructure with adjacent parts of the structure, and subscript $r$ denotes the remaining DOFs outside the substructure. The equations of motion for the substructure are extracted from the full system as 


$$
\left[\begin{array}{ll}
\mathbf{M}_{i j} & \mathbf{M}_{i i}
\end{array}\right]\left\{\begin{array}{l}
\ddot{\mathbf{u}}_{j} \\
\ddot{\mathbf{u}}_{i}
\end{array}\right\}+\left[\begin{array}{ll}
\mathbf{C}_{i j} & \mathbf{C}_{i i}
\end{array}\right]\left\{\begin{array}{l}
\dot{\mathbf{u}}_{j} \\
\dot{\mathbf{u}}_{i}
\end{array}\right\}+\left[\begin{array}{ll}
\mathbf{K}_{i j} & \mathbf{K}_{i i}
\end{array}\right]\left\{\begin{array}{l}
\mathbf{u}_{j} \\
\mathbf{u}_{i}
\end{array}\right\}=\mathbf{P}_{i}
$$

Treating interaction effects at the interface DOFs as interface forces to the substructure [16], the above equation can be rewritten as

$$
\mathbf{M}_{i i} \ddot{\mathbf{u}}_{i}+\mathbf{C}_{i i} \dot{\mathbf{u}}_{i}+\mathbf{K}_{i i} \mathbf{u}_{i}=\mathbf{P}_{i}-\mathbf{M}_{i j} \ddot{\mathbf{u}}_{j}-\mathbf{C}_{i j} \dot{\mathbf{u}}_{j}-\mathbf{K}_{i j} \mathbf{u}_{j}
$$

where the last three terms in the right hand side constitute an interface force vector as input to the substructure.

In order to identify structural parameters of a substructure, the computation of the above interface force vector requires the measurements of all accelerations, velocities and displacements at the interface DOFs [16]. In reality, measurements of accelerations (by accelerometers) are usually preferred over those of velocities and displacements. Therefore, for practicability, to eliminate the requirement of velocities and displacements, Koh et al. [27] proposed the concept of "quasi-static" displacement so as to use only acceleration measurement in the interface force computation. Nonetheless, as mentioned above in the introduction, inaccuracy could result from the inversion of internal stiffness matrix and neglecting the velocity-dependent component.

Note that the identification strategy is model based, i.e. it requires an "a priori" mathematical model of the substructure. The mathematical model is usually part of the finite element model or some simplified model used for design purpose. Thus the parameters identified have physical meanings and can be used directly in the re-analysis by the same model with updated parameters. 


\section{IMPROVED SUBSTRUCTURAL IDENTIFICATION STRATEGY}

In order to improve the accuracy of identification results for substructures, this paper proposes an alternative to make use of acceleration measurement directly in computing the interface force vector. This is done by embedding a simple numerical integration scheme to obtain interface velocity and displacement from measured interface acceleration as shown in the following equations:

$$
\dot{\mathbf{u}}_{j}^{k+1}=\dot{\mathbf{u}}_{j}^{k}+\frac{\Delta t}{2}\left(\ddot{\mathbf{u}}_{j}^{k}+\ddot{\mathbf{u}}_{j}^{k+1}\right) ; \quad \mathbf{u}_{j}^{k+1}=\mathbf{u}_{j}^{k}+\frac{\Delta t}{2}\left(\dot{\mathbf{u}}_{j}^{k}+\dot{\mathbf{u}}_{j}^{k+1}\right)
$$

where $\Delta t$ is the time step. Subscript “ $j$ ” denotes interface DOFs and superscript " $k$ " denotes the step in the time signals.

In reality, noise contamination in measurement signals is inevitable and may result in drift in the integrated velocity and displacement time histories, leading to a low-frequency drift in the interface force vector. Nevertheless, the natural frequencies of a substructure are higher than those of the whole structure; and the dynamic response of a substructure is predominantly excited by force components with their frequencies close to the substructure's frequencies. Therefore, the response from the low-frequency components of the interface force is negligible. Besides, a side benefit of the Sub-SI strategy is that, if there is no internal excitation $\mathbf{P}_{i}$ within a substructure, the strategy is effectively an output-only substructure identification strategy.

The substructure identification strategy is also enhanced through utilizing a recently improved GA as an efficient search engine to identify unknown parameters of substructures. Therefore, this GA-based Sub-SI strategy is a combination of two complementary methods based on two different principles. While the substructural method reduces the identification system size, the improved GA enhances the search effectiveness. 
Recognizing that the search capability of GA depends on the search space, the GA method employed herein integrates an adaptive search space reduction method (SSRM) into an improved GA based on migration and artificial selection (iGAMAS). While SSRM adaptively reduces the search space to expedite the search process, iGAMAS identifies the unknown structural parameters using a multi-feature approach to enhance search diversity from local to global levels [31]. SSRM interacts closely with iGAMAS throughout the search process. Based on the statistical information (mean and standard deviation) of identification results after several runs of iGAMAS, SSRM narrows the search space for those parameters that converge quickly, so as to focus the search effort on the remaining parameters. By progressively and adaptively reducing the limits of the search space, the convergence performance is greatly enhanced.

The multiple features include concurrent evolution of multi-species with different roles, migration between species, artificial selection, regeneration, reintroduction, and a variable data length procedure. The main layout of the multi-feature GA is shown in Figure 2. The robustness of iGAMAS is derived from having multiple species to achieve a good trade off between exploration and exploitation. To achieve this purpose, species $2-4$ use three different mutation operators: random mutation, cyclic non-linear mutation and local nonlinear mutation, respectively, as shown in Figure 2. The main goal of species 2 is to explore the entire search space throughout the search process, while species 3 and 4 focus on exploiting and refining the good solutions in two different ways. In addition, to facilitate cross fertilization, migration operator is employed to exchange individuals between species 2 and 3, as well as between species 3 and 4. An artificial selection operator is utilized to choose the best individual in species $2-4$ through comparison of fitness values, and the best is stored in species 1 . The best individuals in each generation are hence kept in species 1 and they are reintroduced into species 4 for future refinement. Consequently, these multiple features 
greatly enhance the search performance of iGAMAS that can simultaneously explore the whole search space as global search and exploit promising individuals as local search. More details of the multiple features and their functionality can be found in [31].

The improved Sub-SI strategy is schematically shown in Figure 3. For each trial set of unknown parameters, the forward (dynamic) analysis is carried out by numerically solving the dynamic equations given in Eq. (3) for the substructure. The simulated acceleration time history is then directly compared with the measured acceleration time history through a fitness (objective) function. The objective of GA is to adjust the set of trial parameters in order to match the simulated acceleration time histories with the measured ones by minimizing the square error summed across $N_{m}$ measured internal DOFs each with data length $L$ of acceleration time histories. The fitness function, $f_{e}$, is defined as the inverse of the squared error between the measured and simulated (predicted) responses as

$$
f_{e}=\frac{1}{\varepsilon+\sum_{i=1}^{N_{m}} \sum_{j=1}^{L}\left(\ddot{u}_{i, j}^{m}-\ddot{u}_{i, j}^{s}\right)^{2}}
$$

where superscripts $m$ and $s$ denote measured and simulated quantities, respectively. A small value, $\varepsilon$, is used to avoid singularity when the simulated and measured responses happen to match exactly. Its value is chosen to be 0.001 for this study.

\section{NUMERICAL STUDIES}

In order to assess the performance of the proposed strategy, two numerical examples of known-mass and unknown-mass systems are studied. First, a seismically excited system of three buildings linked by two sky-bridges is presented to evaluate the appropriateness of using directly acceleration measurements to account for interaction between substructures. The second example demonstrates the significantly enhanced effectiveness of this strategy on a 
larger structural system of 100-storey shear building with unknown-mass information. For comparison purposes, the CSI strategy is also used to identify the structure as a whole.

The effect of measurement noise is considered at 0,5 , and $10 \%$ noise in the numerical examples. To make a noise-contaminated signal vector, $\mathbf{x}_{\text {con }}$, noise is added to a clean (numerically simulated) signal vector $\mathbf{x}_{c l e}$ as follows:

$$
\mathbf{x}_{\text {con }}=\mathbf{x}_{\text {cle }}+E_{\text {lev }} \times \operatorname{RMS}\left(\mathbf{x}_{\text {cle }}\right) \times \mathbf{N}_{\text {oise }}
$$

where $E_{l e v}$ is a given noise level, $\mathrm{RMS}\left(\mathbf{x}_{\text {cle }}\right)$ root-mean-square value of the clean signal vector, and $\mathbf{N}_{\text {oise }}$ randomly generated noise vector of Gaussian distribution with zero mean and unit standard deviation. To test the search robustness, a fairly broad search range from half to double the exact value is adopted for each unknown parameter. In view of the stochastic nature of GA-based method, identification is repeated 5 times for each case and the average result is presented.

\subsection{Identification of a seismically excited system of three buildings}

The first numerical example considers a system of three shear buildings of 15, 20 and 7 storeys, which are connected by two link bridges as shown in Figure 4(a). This system is simulated to be subjected to the 1940 El Centro earthquake, North-South component. The structural properties for the central building are $m_{1}-m_{11}=7 \times 10^{5} \mathrm{~kg}, m_{12}-m_{20}=5 \times 10^{5} \mathrm{~kg}$, $k_{1}-k_{11}=6 \times 10^{5} \mathrm{kN} / \mathrm{m}$, and $k_{12}-k_{20}=4 \times 10^{5} \mathrm{kN} / \mathrm{m}$. The left and right buildings have $m=6 \times 10^{5} \mathrm{~kg}, k=6 \times 10^{5} \mathrm{kN} / \mathrm{m}$ and $m=4 \times 10^{5} \mathrm{~kg}, k=4 \times 10^{5} \mathrm{kN} / \mathrm{m}$ for all the storeys, respectively. The two link bridges are modelled as linear springs each with horizontal stiffness of $12 \times 10^{5} \mathrm{kN} / \mathrm{m}$. The natural periods of the first two modes of the coupled system are 2.19 and $1.00 \mathrm{~s}$. Rayleigh damping is adopted with damping ratio of $2 \%$ applied to the 
first 2 modes of the coupled system. The response of the entire system to the first $4 \mathrm{~s}$ under the earthquake ground motion is simulated using a time step of $0.001 \mathrm{~s}$. Only acceleration measurement is used as the response quantity for structural identification. The 13 accelerations at levels 0 (ground), 1, 3, 4, 6, 8, 10, 11, 12, 14, 16, 18, and 20 of the central building are used as measurements for the identification.

From the viewpoint of substructuring, the central building can be seen as a substructure of the coupled three-building system. The effect of coupling between the buildings through link bridges on the central building is represented as two coupling forces that apply to the corresponding levels of 4 and 12 of the central building as Figure 4(b), where $F_{1}$ and $F_{2}$ represent the coupling forces from the adjacent buildings. These forces are assumed to be measured and treated as input forces in substructural identification.

While the main focus is to identify the stiffness values, the two damping constants of the central building are also treated as unknowns. The central building is further divided into two substructures: $S_{1}=[11-20]$ (i.e. the $11^{\text {th }}$ to $20^{\text {th }}$ levels inclusive) and $S_{2}=[1-10]$. The results are presented in Table 1 in terms of the absolute mean to absolute maximum errors in the identified stiffness values, in comparison with the results obtained by CSI.

The identified results by the improved Sub-SI strategy are very good. Even in the presence of $10 \%$ noise, the mean error is only about $1 \%$ and the maximum error is less than $3 \%$ of identification results. This demonstrates that the interaction effect at interface DOFs of substructures is appropriately accounted for in the proposed improved Sub-SI strategy using only acceleration measurements.

As seen in Table 1, the improved Sub-SI strategy gives better results than the CSI does. For example, under $10 \%$ noise, the maximum identification error for the CSI is $4.4 \%$ and reduces to $2.4 \%$ for the Sub-SI. Furthermore, the computational time for Sub-SI (224 min. on a duo core 2, 3-GHz PC) is less than that for CSI (276 min.). As an illustration, Figure 5 
presents the results in terms of the ratio of identified stiffness to exact stiffness for the worst case of $10 \%$ noise. The ratios are close to one for all the 20 identified stiffness values, illustrating the high accuracy of the proposed Sub-SI strategy.

\subsection{Identification of 100-DOF unknown-mass system}

To further assess the performance of the proposed strategy, this example involving a much larger system tackles a greater challenge whereby not only stiffness and damping but also mass parameters are to be simultaneously identified. Note that many Sub-SI methods based on state-space formulation or frequency domain are not applicable to identification of unknown-mass structural systems [16, 25].

Here, a larger structural system of a 100-storey shear building is to be identified. Its structural properties are $m_{1}-m_{30}=15 \times 10^{5} \mathrm{~kg}, m_{31}-m_{60}=12 \times 10^{5} \mathrm{~kg}, m_{61}-m_{100}=8 \times 10^{5}$ $\mathrm{kg}, k_{1}-k_{30}=16 \times 10^{5} \mathrm{kN} / \mathrm{m}, k_{31}-k_{60}=12 \times 10^{5} \mathrm{kN} / \mathrm{m}$, and $k_{61}-k_{100}=9 \times 10^{5} \mathrm{kN} / \mathrm{m}$. The natural periods of the first two modes are 10.5 and $4.1 \mathrm{~s}$. Damping ratio is $2 \%$ for the first two modes. Two input forces are applied at the $3^{\text {rd }}$ and $8^{\text {th }}$ nodes of every 10 floor levels. The measured accelerations of the building are numerically generated using a time step of $0.001 \mathrm{~s}$. Six accelerations at the $1^{\text {st }}, 3^{\text {rd }}, 5^{\text {th }}, 6^{\text {th }}, 8^{\text {th }}$ and $10^{\text {th }}$ nodes of every 10 storeys $(60 \%$ of DOFs) are extracted for use in the identification. The complete structure is divided into 20 substructures: $S_{1}=[100-96], S_{2}=[96-91], \ldots S_{20}=[6-1]$.

The absolute errors of identified stiffness and mass values are summarized in Table 2 and Table 3, respectively. Nearly exact identification results using the proposed improved strategy can be obtained in the noise-free case. The proposed strategy is shown to be able to identify such a large structure with incomplete measurement, achieving mean error of less than $3 \%$ even for $10 \%$ noise. This is a remarkable feat that, to the knowledge of the authors, has not been reported thus far. The results are also better than those obtained by two recent Sub-SI methods that identify a structural system of 50-DOFs for $5 \%$ noise [23, 27], even 
though a larger system of 100 DOFs is studied here. The mean error of about $1 \%$ of stiffness results achieved by the proposed strategy is much smaller than that of $5.1 \%$ and $10.5 \%$ using two different Sub-SI methods that made use of relative acceleration measurements to account for interaction effects $[23,27]$. Likewise for identified mass results in Table 3, the error of about $1 \%$ using the proposed strategy is better than that of $5 \%$ using a previous GA-based Sub-SI method [27]. This comparison demonstrates that the multi-feature GA adopted in the proposed strategy achieves more accurate results than the GA algorithm used in the recent Sub-SI methods. The results for this unknown-mass system also confirm that the proposed strategy is appropriate in accounting for the interaction effect.

For this unknown-mass structural system with 202 unknowns in total, the Sub-SI strategy yields much better identified results than the CSI one does. In stiffness identification, as shown in Table 2, in the presence of $10 \%$ noise the mean error of identified results for CSI is about $6.91 \%$ and reduces to $2.97 \%$ for Sub-SI. Correspondingly, the maximum absolute error reduces from $17.99 \%$ to $9.75 \%$. Likewise, in mass identification, there is a significant improvement as shown in Table 3. In addition to improvement in accuracy, the proposed strategy is more efficient with $27 \%$ saving of overall computational time as compared to CSI (391 min. on a duo core 2, 3-GHz PC).

\section{EXPERIMENTAL STUDIES}

Although a number of Sub-SI methods have recently been proposed in similar research as discussed in the literature review, few of them have been verified experimentally, including those dealing with identification of mass and stiffness parameters simultaneously. The proposed strategy is tested by an experimental study involving a small-scale steel frame with a total height of $2.0 \mathrm{~m}$ and a plan of $0.2 \times 0.4 \mathrm{~m}$. The 10 -storey frame, as shown in Figure 6 , is designed with 6 columns (rectangular cross section of $4.5 \times 25 \mathrm{~mm}$ ) and relatively stiff beam (square hollow sections of $25 \times 25 \times 3 \mathrm{~mm}$ ). By the symmetry of the structure and loading, the 
significant motion is a uni-directional translation at each level. Accounting for beam and column masses, the lumped mass values for levels 1 to 9 and level 10 are $3.25 \mathrm{~kg}$ and $3.00 \mathrm{~kg}$, respectively. Impact test shows that the natural frequencies of the frame are from $8.3 \mathrm{~Hz}$ for the first mode to $115.23 \mathrm{~Hz}$ for the tenth mode.

The analytical model does not necessarily represent the real model in terms of stiffness due to modelling error $[14,32]$. In order to verify the identification results using the improved Sub-SI strategy, the actual storey stiffness values are measured by static experiments and used as benchmark for comparison.

\subsection{Static test}

Static test is conducted to estimate of the as-built stiffness of the frame structure. The model is mounted horizontally to a rigid vertical support to facilitate applying force at each level by hanging weights as shown in Figure 6. To compute the stiffness value for each level, the difference of deflections between two adjacent storeys is measured by displacement transducers. Figure 7 shows that for weights from 20 to $35 \mathrm{~kg}$, the stiffness values at all levels are very consistent while for the weight of $10 \mathrm{~kg}$, the stiffness values have a small fluctuation at levels 4 and 6 . Thus, the average stiffness values corresponding to the weights of 20 to $35 \mathrm{~kg}$ are presented in Table 4. These measured stiffness values are used as benchmark values for comparing with identified results obtained from dynamic test.

\subsection{Dynamic test}

Dynamic test set-up in laboratory is shown in Figure 8. The force generated by the shaker vertically acts on the steel frame by a connection rod at level 10 . Excitation forces are input into signal generator (Signametrics function/pulse generator, model SM-1020). The force signal is then passed through a power amplifier in order to produce sufficient power for the electromagnetic shaker (Labworks ET-126B). To enhance the reliability of identification results, four different force inputs (labelled A, B, C, D) are generated by 1000 data points at a 
time step of $0.0001 \mathrm{~s}$. Hence only a short duration $(0.1 \mathrm{~s})$ of measurement is needed. These force time histories are measured by an Integrated Circuit Piezoelectric (ICP) force sensor (PCB-208C02) and are shown in Figure 9. In general, to achieve accurate identification results, high signal-to-noise ratio is required of the measured response and this would depend on the excitation level and quality of sensors.

The acceleration responses of the frame are measured using 10 accelerometers mounted at upper plane of each storey. The signals from the force sensor and the accelerometers are passed through signal conditioners and recorded using a 16-channel digital oscilloscope (Yokogawa-DL716E) at a sampling rate of 10,000 points/s. Although the highest frequency of the frame corresponding to the $10^{\text {th }}$ mode is $115.23 \mathrm{~Hz}$, the high sampling frequency allows for a more accurate simulation of the response during identification since the frequencies of a substructure are often higher than those of the complete structure, and the accuracy of interface acceleration affects the identification results of a substructure.

Acceleration time histories of $0.1 \mathrm{~s}$, starting from immediately before the application of force on the frame, are extracted as input to the identification strategy. The signals are processed by removing any mean offset that may exist in the raw data. This is done by computing the mean of 1000 sample data points shortly prior to applying forces. The noise level estimated by determining the ratio of the standard deviation of in the pre-excitation signal to that of the 1000 data points in the post-excitation signal. The ratio indicates that the noise level ranges from 1 to 5\% in all signals. Further details can be referred to [33]

\subsection{Identification with complete measurements}

Identification of unknown-mass systems is much more difficult compared to systems where the mass is assumed known. First, identification is carried out using the complete measurements, i.e. one force and ten acceleration measurements. The structural model used for identification is idealised as a shear building model with Rayleigh damping. The search 
limits for all case are set as 300-600 kN/m for all stiffness parameters, 2.5-3.5 $\mathrm{kg}$ for all mass parameters, and 0-4 and 0-0.0002 for two damping parameters, respectively. The identification procedure is carried out five times for each input force.

To assess the performance of the improved Sub-SI strategy for various substructures, three substructures are studied as shown in Figure 10. In substructure $1, S_{1}=[1-3]$, and substructure 2, $S_{2}=[3-9]$, no force is applied, whereas in substructure $3, S_{3}=[9-10]$, a dynamic force is applied at level 10. In this example, the substructures have overlapping levels, providing an alternative to the non-overlap version of substructural identification used in the previous two examples. The CSI strategy is also adopted to identify all structural parameters of this frame and its results are compared with those of the Sub-SI strategy. For fair comparison, the same improved GA method is employed with the same number of evaluations for both strategies so that their computational times are the same.

The identification for the frame is carried out for four different forces and their stiffness results are shown in Table 5, along with the mean and the standard deviation values. The results show that the improved strategy yields very similar stiffness values for different input forces. The maximum ratio of standard deviation to mean values is only 0.065 , showing the consistency of the identification results obtained for different input forces.

The mean stiffness results using the improved Sub-SI strategy (as shown in Table 5) and the CSI strategy are compared with the measured values based on the static test in Table 4. The corresponding error is computed by using the measured stiffness values in the static test as the baseline. The identified mass results of Sub-SI and CSI are shown in Table 6, where the error is computed by considering the estimated mass (as discussed earlier in this section) as the baseline.

Table 4 shows that the identified results from the Sub-SI strategy are excellent. To be able to experimentally identify the structural stiffness with unknown-mass with mean error of 
less than $6 \%$ and maximum error of less than $9 \%$ for different substructures is a notable accomplishment. An important point to note here is the achievement of good identification results in substructures 1 and 2 even with no applied force within the substructure. This demonstrates that the Sub-SI strategy can be used advantageously and effectively as outputonly substructural identification.

It is observed in Table 4 that for the CSI strategy, an error of more than $29 \%$ at level 1 is much greater than those at the other storeys. From the viewpoint of structural identification, the structural part near the boundary (or support), i.e. level 1 , is often very difficult to identify accurately. This can be attributed to the difficulty in modelling the boundary condition precisely in the numerical model. Furthermore, the vibration response at this level near the fixed support contributes less to the identification as compared to higher levels in the structure. Therefore, if the whole structure is identified at one go, the identification result of this storey is often less accurate. The proposed Sub-SI strategy deals with this difficulty by treating the boundary as an interface DOF of substructure $S_{1}$. In addition, all measured responses within $S_{1}$ have roughly the same contribution to the fitness value in the objective function that plays a crucial role in the GA-based identification procedure. As a result, the stiffness of level 1 in $S_{1}$ is identified with better accuracy (maximum error of about $8 \%$ as compared to about $30 \%$ by CSI). The stiffness identification results using CSI and Sub-SI are compared in Figure 11 where static test results are treated as the benchmark (correct) values. It is seen that the proposed Sub-SI strategy gives better results than the CSI strategy does with a reduction of mean error from $12.48 \%$ to $5.72 \%$ accordingly. Likewise, Table 6 shows that the identified mass results of the improved Sub-SI strategy are also better than those of the CSI strategy when compared to the mass values computed based on known density and geometric dimensions. 
Note that some levels are included in two substructures, known as the overlap levels. For example, level 3 is an overlap between substructures 1 and 2; similarly level 9 between substructures 2 and 3. For such overlap levels, it is of great interest to check the consistency of stiffness values obtained from different substructures. As seen in Table 4, for the overlap level 3, the identified stiffness value of approximately $416.4 \mathrm{kN} / \mathrm{m}$ obtained in $S_{1}$ is almost the same as approximately $410.2 \mathrm{kN} / \mathrm{m}$ in $S_{2}$ (i.e. $1.5 \%$ difference). Similarly, for the overlap level 9, the identified stiffness value of $374.8 \mathrm{kN} / \mathrm{m}$ in $S_{2}$ is nearly equal to $373.1 \mathrm{kN} / \mathrm{m}$ in $S_{3}$ ( $0.4 \%$ difference). Therefore, it is evident that the proposed Sub-SI strategy is consistent in identification results based on different substructures.

\subsection{Identification with incomplete measurements}

The performance of the improved strategy is further examined through the identification of substructure 2 with incomplete acceleration measurements. Two incomplete measurement cases corresponding to 7 and 4 available sensors are conducted. For the 7-sensor case, the sensors are located at levels 2, 3, 4, 6, 7, 8, and 9. For the 4-sensor case, the sensors are placed at levels 2, 4, 8, and 9. The Sub-SI strategy based on incomplete measurement is compared with the CSI strategy based on complete measurement in Table 7. In spite of the presence of inevitable experimental and numerical errors, the improved Sub-SI strategy is able to identify a substructure with only using 4 sensors with a mean error of less than $4 \%$, better than the error of about $11 \%$ for the CSI strategy using 8 sensors . The results generally show that the proposed Sub-SI strategy improves the accuracy of identification results even using a lesser number of sensors.

\section{CONCLUSIONS}

In this paper, an improved substructural identification (Sub-SI) strategy for large structural systems is presented. The following conclusions can be drawn: 
1. The combined use of substructural identification idea and multi-feature GA allows the proposed strategy to identify not only stiffness and damping parameters, but also mass parameters for large structural systems.

2. The strategy has improved the accuracy of identification results as compared to some recent substructural identification studies. This demonstrates that the use of interface acceleration measurements directly to account for the interaction effects for substructures is justified.

3. The better performance of the proposed strategy than that of the complete structural identification strategy is numerically demonstrated and further experimentally verified using a 10-storey laboratory-scale steel frame.

4. Since substructures can be identified independently of one another, the identification of a substructure is not affected by factors outside it, including applied force, noise, boundary conditions and incomplete information in other substructures. This is applicable for local damage detection of critical parts in large structural systems, without having to involve the whole structure.

5. The strategy also permits output-only identification of a particular substructure, as demonstrated in the experimental study for substructures 1 and 2.

\section{ACKNOWLEDGEMENT}

The first author gratefully acknowledges the research scholarship provided by the National University of Singapore for his Ph.D. study. The authors are grateful to Prof. Ian F. C. Smith at Applied Computing and Mechanics Laboratory, EPFL for his useful comments. The technical assistance rendered by Ms. A. Tan and Mr. K. B. Rasman in the experimental study is also greatly appreciated. 


\section{REFERENCES}

1. Karbhari VM and Ansari F. Structural health monitoring of civil infrastructure systems. CRC Press, 2009.

2. Spencer Jr BF, Ruiz-Sandoval ME, and Kurata N. Smart sensing technology: Opportunities and challenges. Structural Control and Health Monitoring 2004; 11(4): 349-368.

3. Glaser SD, Li H, Wang ML, Ou J, and Lynch J. Sensor technology innovation for the advancement of structural health monitoring: a strategic program of US-China research for the next decade. Smart Structures and Systems 2007; 3(2).

4. Perry MJ, Koh CG, and Choo YS. Modified genetic algorithm strategy for structural identification. Computers and Structures 2006; 84(8-9): 529-540.

5. Hoshiya $\mathrm{M}$ and Saito E. Structural Identification by Extended Kalman Filter. Journal of Engineering Mechanics 1984; 110(12): 1757-1770.

6. Ghanem R and Shinozuka M. Structural-system identification. I: Theory. Journal of Engineering Mechanics 1995; 121(2): 255-264.

7. Carden EP. Vibration Based Condition Monitoring: A Review. Structural Health Monitoring 2004; 3(4): 355-377.

8. Xu B, Wu Z, Chen G, and Yokoyama K. Direct identification of structural parameters from dynamic responses with neural networks. Engineering Applications of Artificial Intelligence 2004; 17(8): 931-943.

9. Perry MJ and Koh CG. Output-only structural identification in time domain: Numerical and experimental studies. Earthquake Engineering and Structural Dynamics 2008; 37(4): 517-533.

10. Robert-Nicoud Y, Raphael B, Burdet O, and Smith IFC. Model identification of bridges using measurement data. Computer-Aided Civil and Infrastructure Engineering 2005; 20(2): 118-131.

11. Saitta S, Kripakaran P, Raphael B, and Smith IFC. Improving system identification using clustering. Journal of Computing in Civil Engineering 2008; 22(5): 292-302.

12. Smith IFC and Saitta S. Improving knowledge of structural system behavior through multiple models. Journal of Structural Engineering 2008; 134(4): 553-561.

13. Saitta S, Raphael B, and Smith IFC. Data mining techniques for improving the reliability of system identification. Advanced Engineering Informatics 2005; 19(4): 289-298.

14. Catbas FN, Ciloglu SK, Hasancebi O, Grimmelsman K, and Aktan AE. Limitations in Structural Identification of Large Constructed Structures. Journal of Structural Engineering 2007; 133(8): 1051-1066.

15. Spencer JBF, Nagayama T. Smart sensor technology: a new paradigm for structural health monitoring. Proceedings of Asia-Pacific Workshop on Structural Health Monitoring, Yokohama, Japan 2006.

16. Koh CG, See LM, and Balendra T. Estimation of structural parameters in time domain. A substructure approach. Earthquake Engineering \& Structural Dynamics 1991; 20(8): 787-801.

17. Yun C-B and Lee H-J. Damage estimation using substructural identification in time domain. Worcester, MA, USA: ASCE. 1996.

18. Lee C-G and Yun C-B. Parameter identification of linear structural dynamic systems. Computers and Structures 1991; 40(6): 1475-1487.

19. Goodwin GC and Sin KS. Adaptive filtering prediction and control. Prentice-Hall: Englewood Cliffs, N.J., 1984. 
20. Tee KF, Koh CG, and Quek ST. Substructural first- and second-order model identification for structural damage assessment. Earthquake Engineering and Structural Dynamics 2005; 34(15): 1755-1775.

21. Juang JN, Phan M, Horta LG, and Longman RW. Identification of observer/Kalman filter Markov parameters: theory and experiments. Journal of Guidance, Control, and Dynamics 1993; 16(2): 320-329.

22. Juang JN and Pappa RS. An eigensystem realization algorithm for modal parameter identification and model reduction. Journal of Guidance, Control, and Dynamics 1985; 8(5): 620-627.

23. Tee KF, Koh CG, and Quek ST. Numerical and Experimental Studies of a Substructural Identification Strategy. Structural Health Monitoring 2009; 8(5).

24. Koh CG, Tee KF, and Quek ST. Condensed model identification and recovery for structural damage assessment. Journal of Structural Engineering 2006; 132(12): 2018-2026.

25. Yun C-B and Bahng EY. Substructural identification using neural networks. Computers and Structures 2000; 77(1): 41-52.

26. $\mathrm{Xu} \mathrm{B}$ and $\mathrm{Du} \mathrm{T}$. Direct substructural identification methodology using acceleration measurements with neural networks. San Diego, CA, United States: Proceedings of SPIE - The International Society for Optical Engineering. 2006.

27. Koh CG, Hong B, and Liaw CY. Substructural and progressive structural identification methods. Engineering Structures 2003; 25(12): 1551-1563.

28. Hongwei H and Yang JN. Damage identification of substructure for local health monitoring. Smart Structures and Systems 2008; 4(Copyright 2009, The Institution of Engineering and Technology): 795-807.

29. Sandesh S and Shankar K. Time Domain Parametric Identification of Plate Bending Rigidity Coefficients using Substructural Approach. in 2nd International Congress on Computational Mechanics and Simulation (ICCMS-06). IIT Guwahati, India. 2006.

30. Koh CG and Thanh TN, Challenges and Strategies in Using Genetic Algorithms for Structural Identification, in Soft Computing in Civil and Structural Engineering, B.H.V. Topping and Y. Tsompanakis, Editors. 2009, Saxe-Coburg Publications: Stirlingshire, UK. p. 203-226.

31. Koh CG and Perry MJ. Structural Identification and Damage Detection using Genetic Algorithms. Taylor \& Francis: London, UK, 2010.

32. Raphael B and Smith IFC. Computer-Aided Engineering. Wiley, 2003.

33. Trinh TN. Evolutionary Divide-And-Conquer Strategy for Identification of Structural Systems and Moving Forces. Ph.D. thesis, National University of Singapore. 2010. 


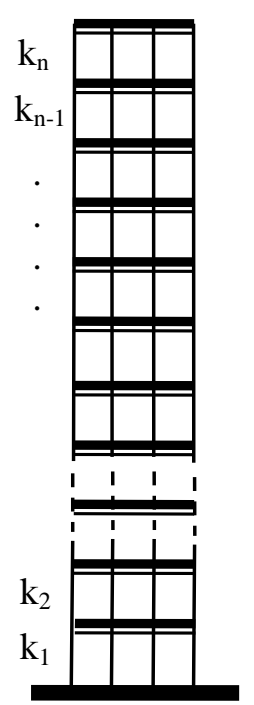

(a)

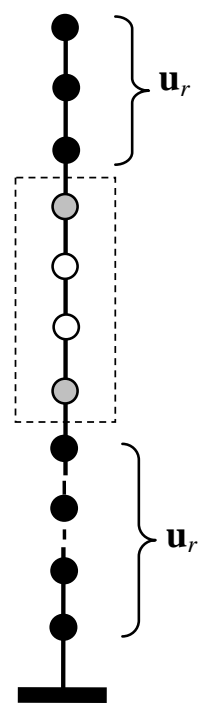

(b)

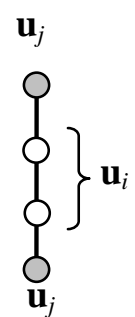

Internal DOFs (i)

Interface DOFs $(j)$

- Remaining DOFs $(r)$

(c)

Figure 1. (a) Shear building; (b) lumped-mass system; (c) a substructure.

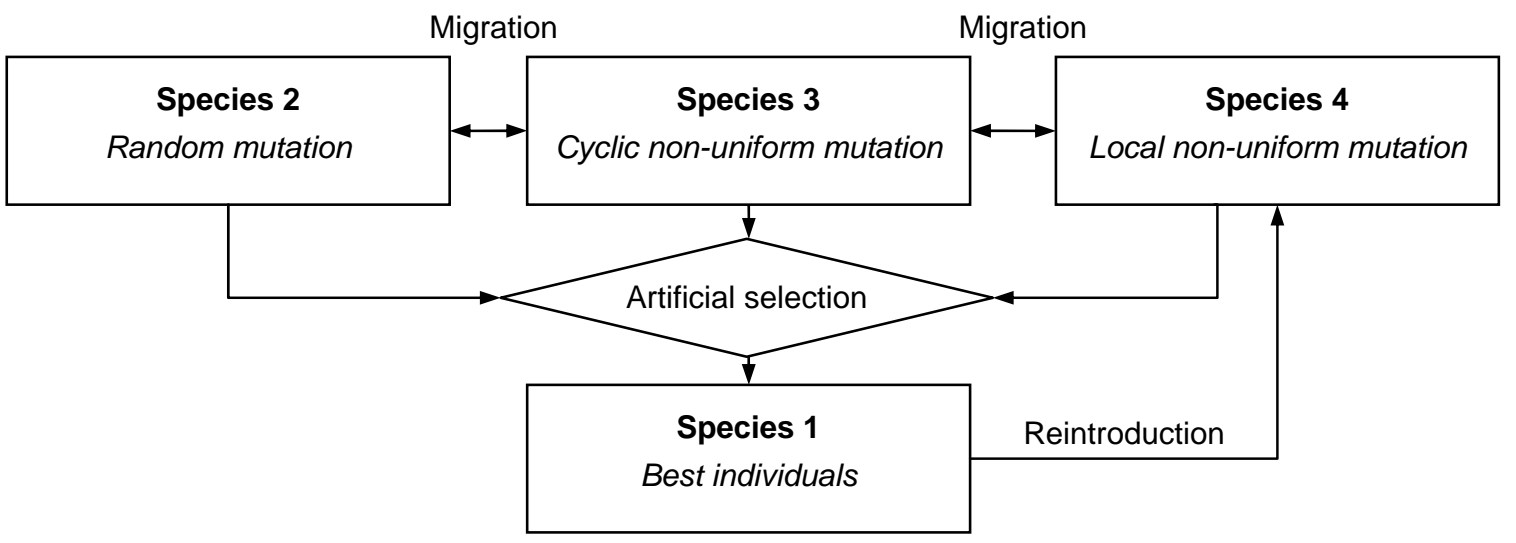

Figure 2. Layout of a multi-feature GA with 4 species using three different mutation operators. 


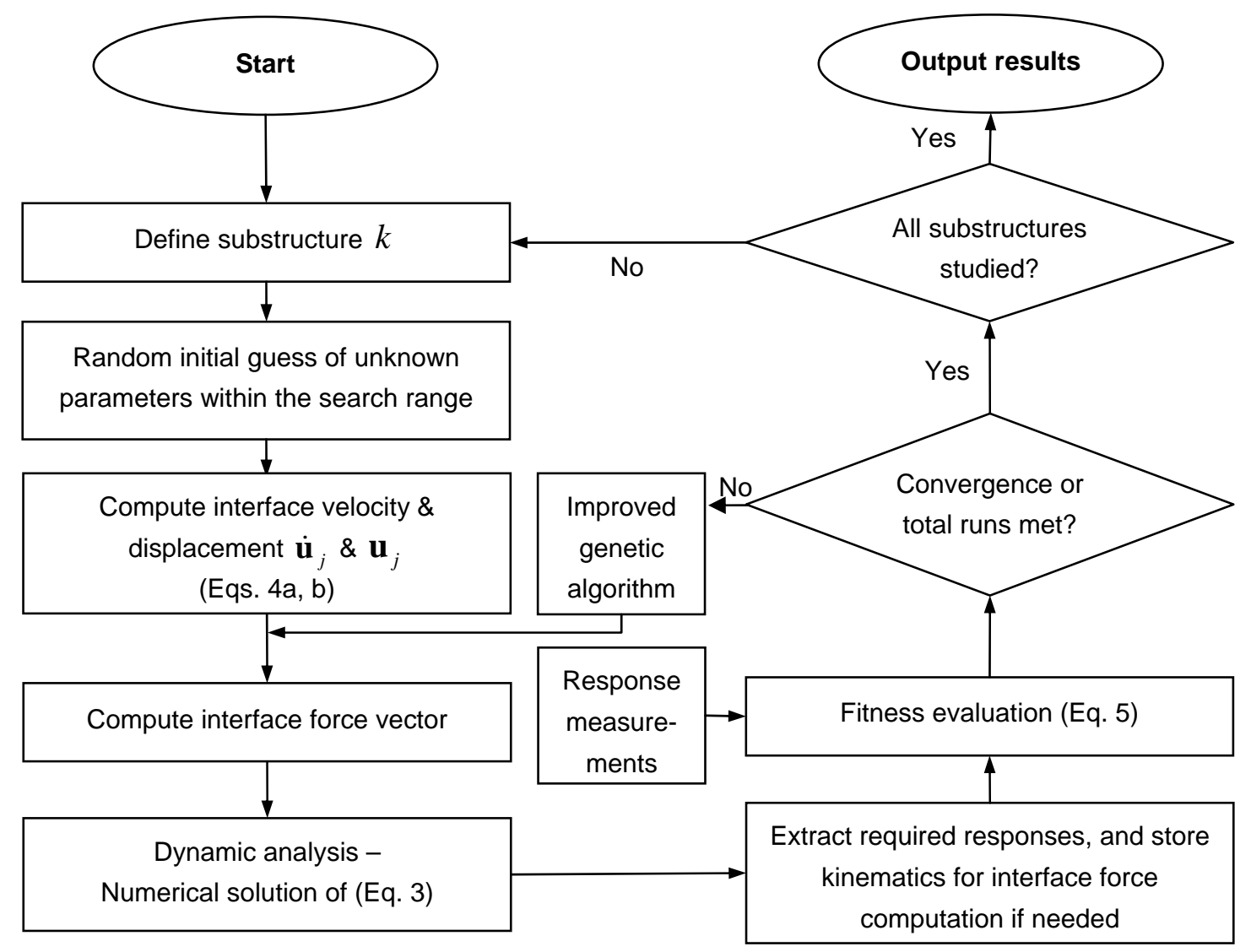

Figure 3. Block diagram of the improved Sub-SI strategy. 
(a)

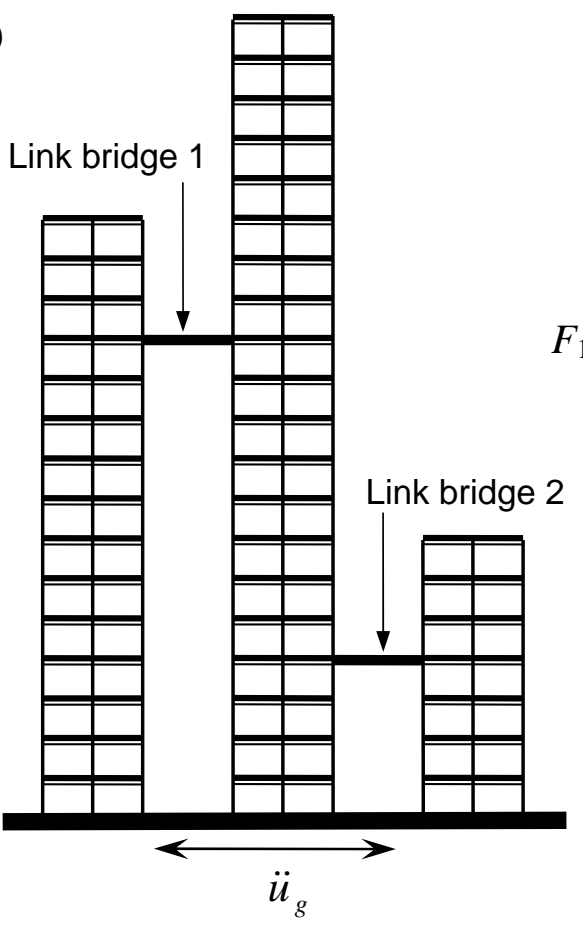

(b)

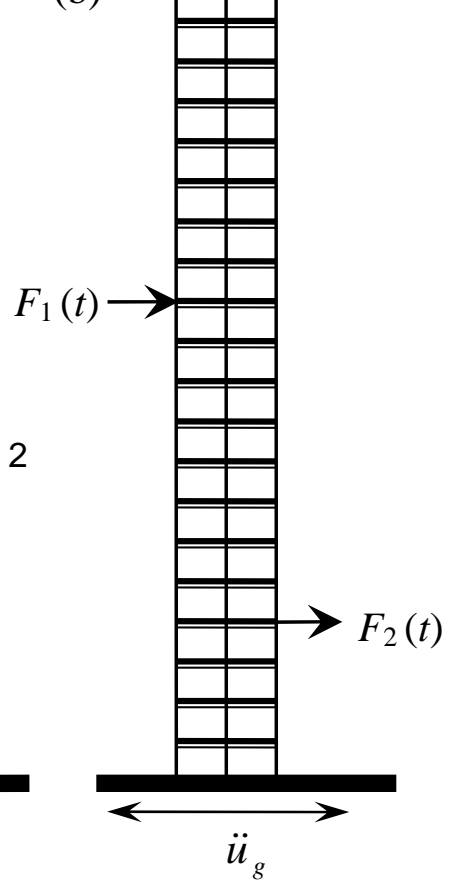

Figure 4. (a) Three connected buildings; (b) central building for identification.

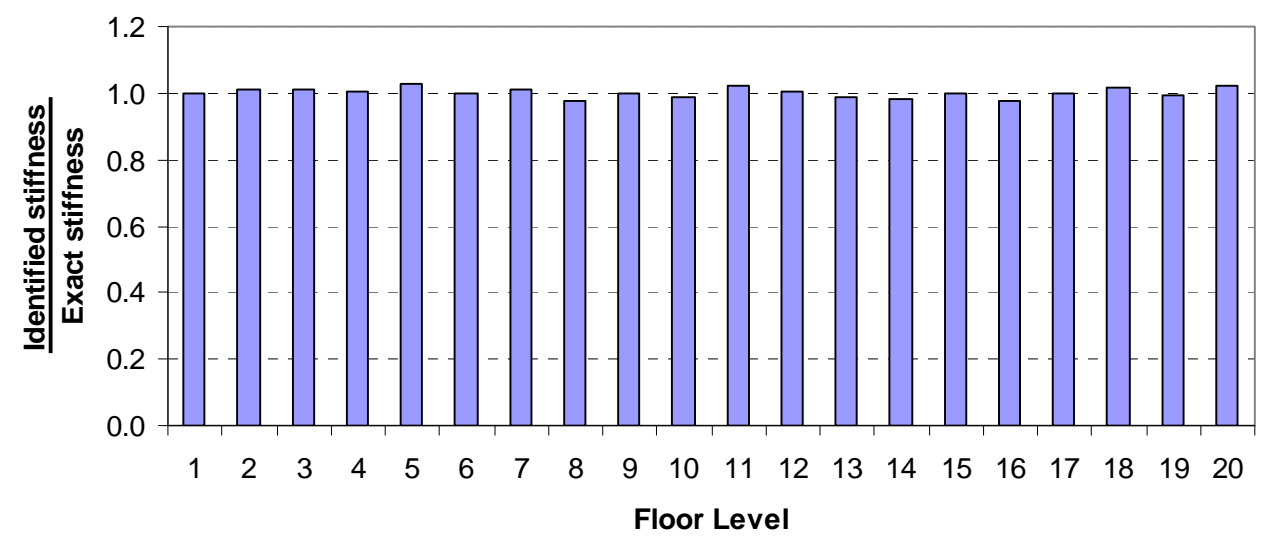

Figure 5. Ratio of identified stiffness to exact stiffness using the improved Sub-SI strategy for $10 \%$ noise. 


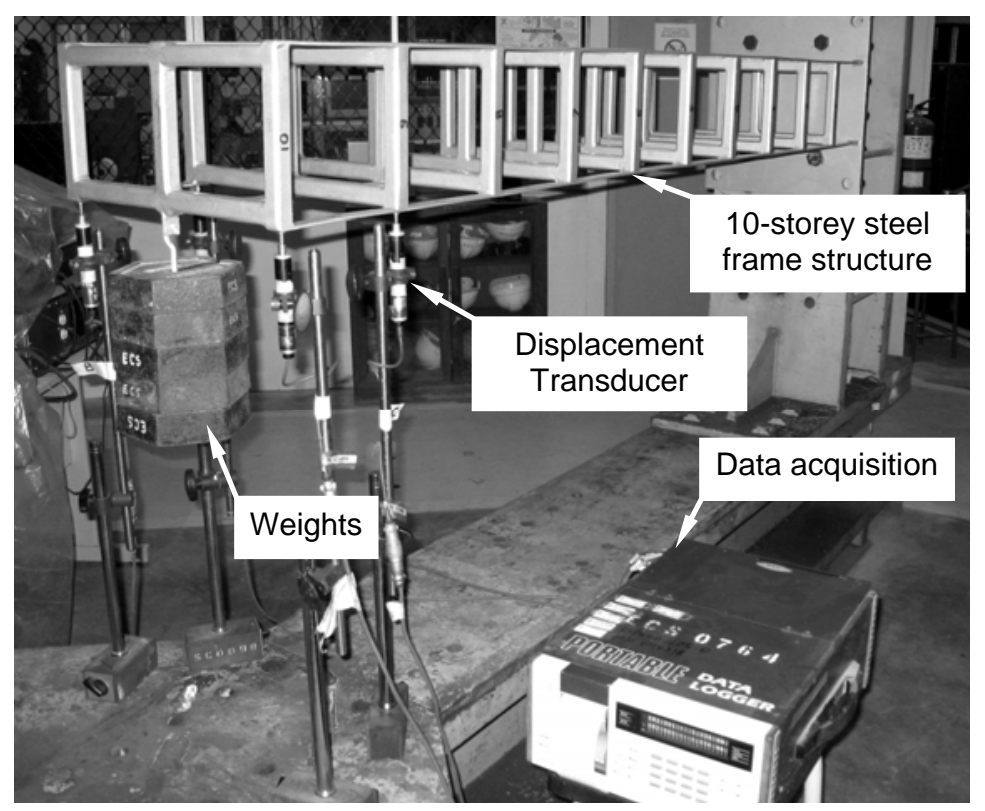

Figure 6. Static test set-up.

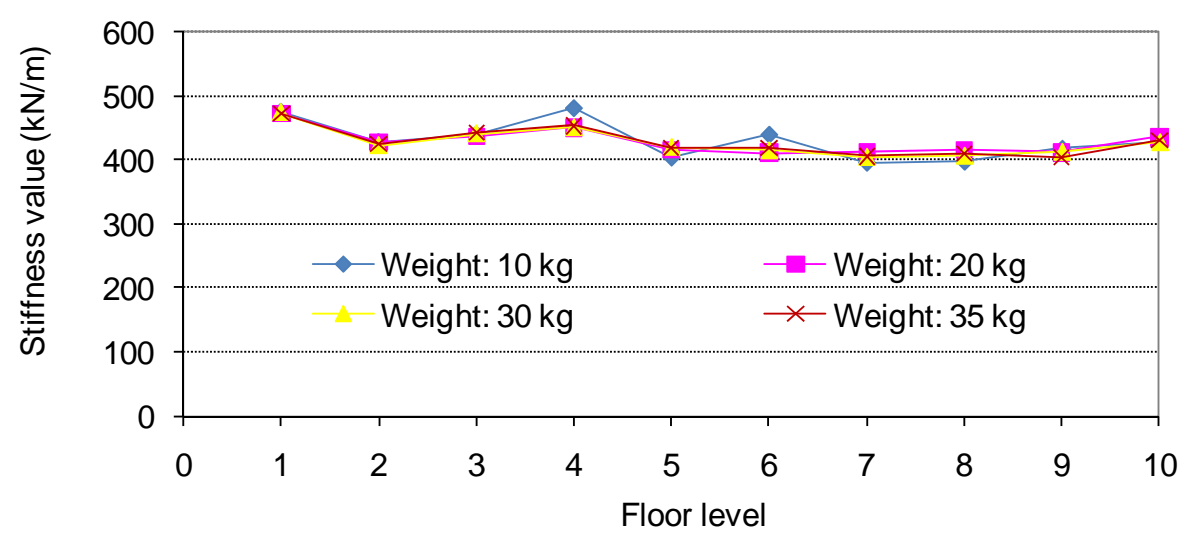

Figure 7. Stiffness for each level obtained for different weights in the static test. 


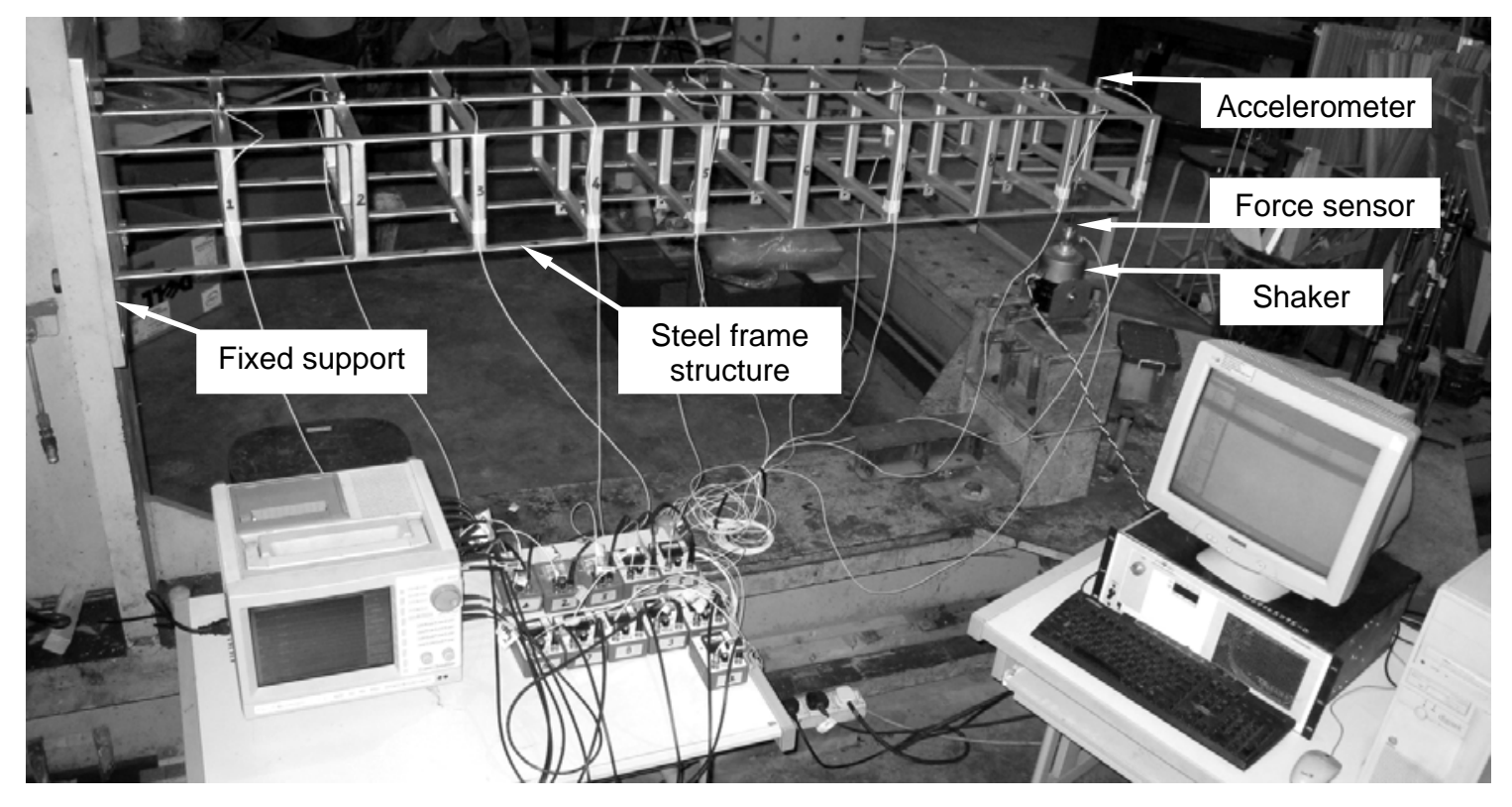

Figure 8. Dynamic test set-up.
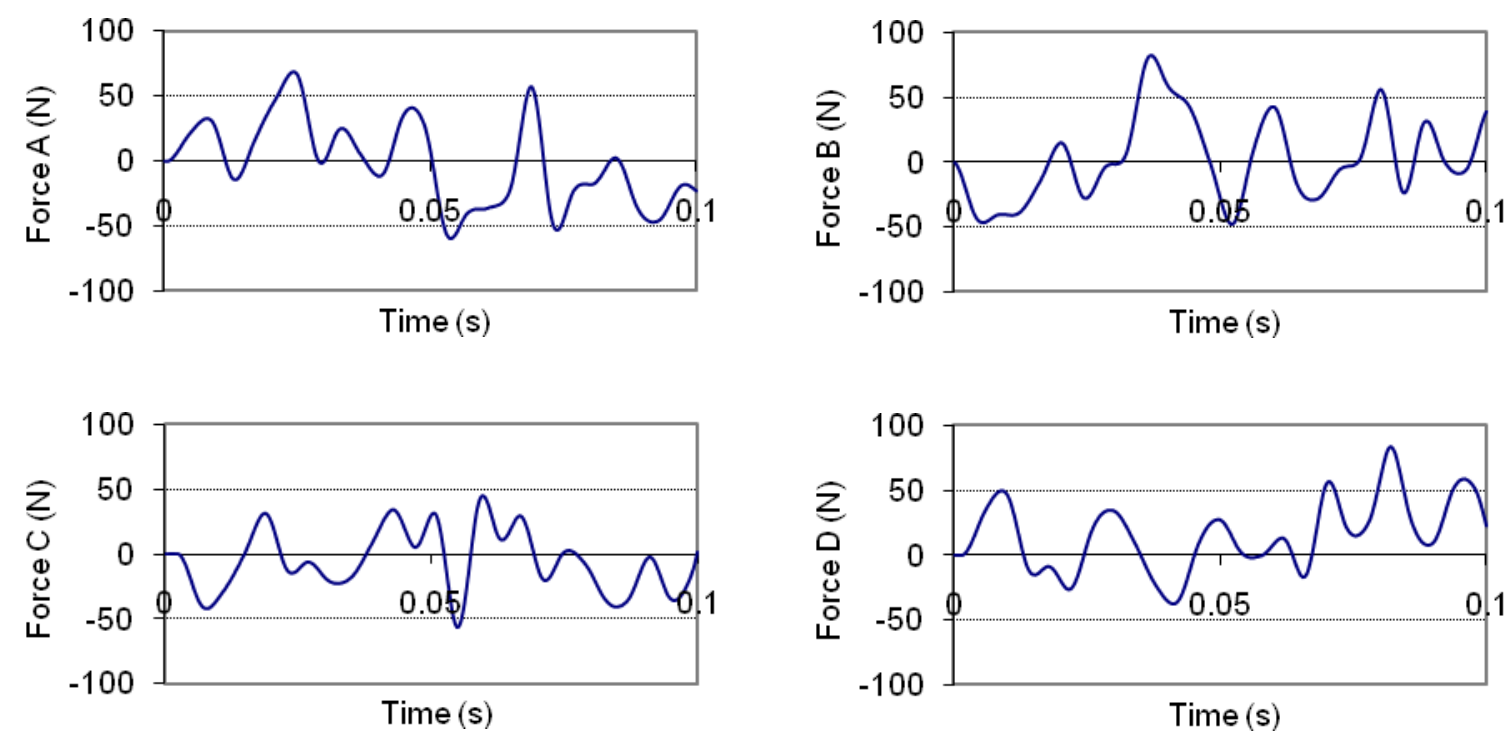

Figure 9. Time histories of four measured forces used in the experiment. 


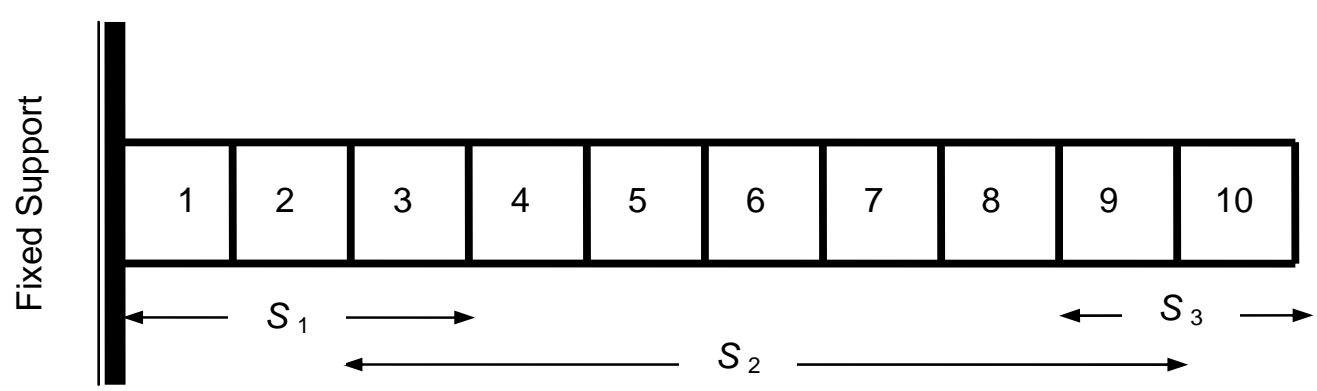

Figure 10. Three substructures to be identified in the experimental study.

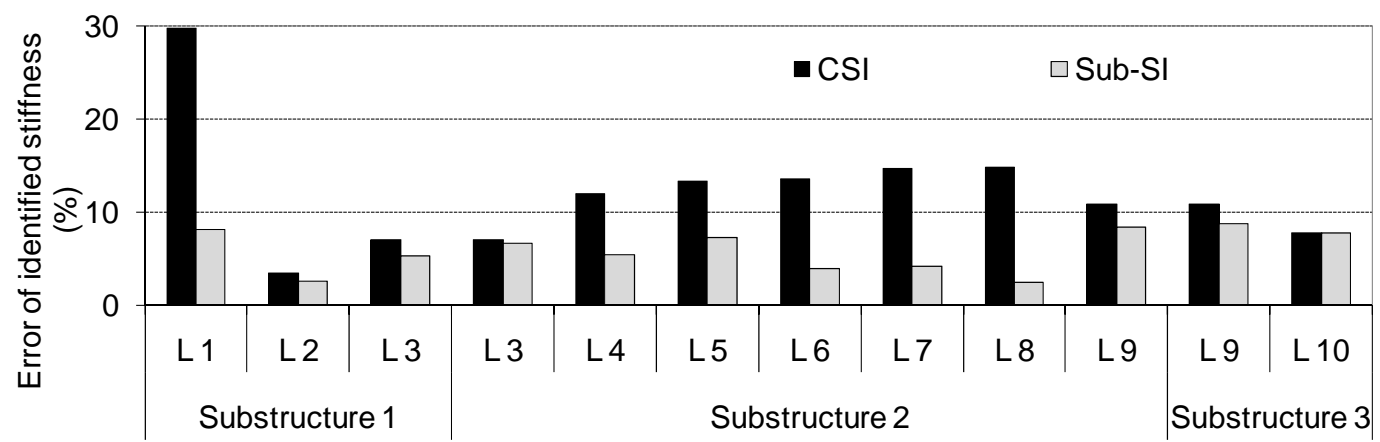

Figure 11. Errors of the stiffness results identified using complete structural identification (CSI) and substructure identification (Sub-SI) strategy in the experimental study. 
Table 1. Absolute errors of identified stiffness values of the central 20-storey building.

\begin{tabular}{ccccccc}
\hline \multirow{2}{*}{ Noise level (\%) } & \multicolumn{2}{c}{ Mean error (\%) } & & \multicolumn{2}{c}{ Maximum error (\%) } \\
\cline { 2 - 3 } \cline { 5 - 6 } & CSI & Sub-SI & & CSI & Sub-SI \\
\hline 0 & 0.04 & 0.01 & & 0.09 & 0.03 \\
5 & 0.72 & 0.57 & & 2.98 & 1.93 \\
10 & 1.56 & 1.01 & & 4.40 & 2.44 \\
\hline
\end{tabular}

Table 2. Absolute errors of identified stiffness values of 100-DOF unknown-mass system.

\begin{tabular}{cccccc}
\hline \multirow{2}{*}{ Noise level (\%) } & \multicolumn{2}{c}{ Mean error (\%) } & & \multicolumn{2}{c}{ Maximum error (\%) } \\
\cline { 2 - 3 } \cline { 5 - 6 } & CSI & Sub-SI & & CSI & Sub-SI \\
\hline 0 & 4.80 & 0.00 & & 13.15 & 0.07 \\
5 & 5.76 & 0.95 & & 15.59 & 2.99 \\
& & $\left(5.10^{*}\right)$ & & & \\
10 & 6.91 & $\left(10.46^{* *}\right)$ & & & 17.99 \\
\hline
\end{tabular}

* Results for a 50-DOF unknown-mass system based on a Sub-SI method that used relative acceleration measurements to account for interaction effects and adopted the genetic algorithm (GA) to identify structural parameters [27].

** Results for a 50-DOF known-mass system based on a substructural first- and second-order model identification method that used relative acceleration measurements to account for interaction effects and adopted the eigensystem realization algorithm (ERA) together with the observer/Kalman filter identification (OKID) to identify structural parameters [23].

Table 3. Absolute errors of identified mass values of 100-DOF unknown-mass system.

\begin{tabular}{ccccccc}
\hline \multirow{2}{*}{ Noise level (\%) } & \multicolumn{2}{c}{ Mean error (\%) } & & \multicolumn{2}{c}{ Maximum error (\%) } \\
\cline { 2 - 3 } \cline { 5 - 6 } & CSI & Sub-SI & & CSI & Sub-SI \\
\hline 0 & 3.36 & 0.00 & & 14.67 & 0.07 \\
5 & 3.79 & 0.81 & & 13.69 & 3.91 \\
10 & & $\left(5.00^{*}\right)$ & & & \\
& 4.71 & 2.50 & & 14.45 & 8.24 \\
\hline
\end{tabular}

* Results for a 50-DOF unknown-mass system based on a Sub-SI method that used relative acceleration measurements to account for interaction effects and adopted the genetic algorithm (GA) to identify structural parameters [27]. 
Table 4. Storey stiffness values and identification errors of the 10-storey frame based on complete measurements in the experimental study.

\begin{tabular}{|c|c|c|c|c|c|c|}
\hline \multirow[b]{2}{*}{ Substructure } & \multirow[b]{2}{*}{$\begin{array}{l}\text { Floor } \\
\text { level }\end{array}$} & \multirow{2}{*}{$\begin{array}{c}\text { Static } \\
\text { Measured } \\
\text { stiffness } \\
(\mathrm{kN} / \mathrm{m}) \\
\end{array}$} & \multicolumn{2}{|c|}{ CSI } & \multicolumn{2}{|c|}{ Sub-SI } \\
\hline & & & $\begin{array}{c}\text { Identified } \\
\text { stiffness } \\
(\mathrm{kN} / \mathrm{m}) \\
\end{array}$ & $\begin{array}{c}\text { Absolute } \\
\text { error } \\
(\%) \\
\end{array}$ & $\begin{array}{c}\text { Identified } \\
\text { stiffness } \\
(\mathrm{kN} / \mathrm{m}) \\
\end{array}$ & $\begin{array}{c}\text { Absolute } \\
\text { error } \\
(\%) \\
\end{array}$ \\
\hline \multirow[t]{3}{*}{1} & 1 & 472.78 & 332.15 & 29.75 & 434.50 & 8.10 \\
\hline & 2 & 424.38 & 409.64 & 3.47 & 435.33 & 2.58 \\
\hline & 3 & 439.60 & 408.73 & 7.02 & 416.44 & 5.27 \\
\hline \multirow[t]{7}{*}{2} & 3 & 439.60 & 408.73 & 7.02 & 410.19 & 6.69 \\
\hline & 4 & 450.89 & 396.84 & 11.99 & 426.59 & 5.39 \\
\hline & 5 & 425.70 & 368.95 & 13.33 & 394.71 & 7.28 \\
\hline & 6 & 414.48 & 358.35 & 13.54 & 398.21 & 3.92 \\
\hline & 7 & 399.15 & 340.69 & 14.65 & 382.75 & 4.11 \\
\hline & 8 & 408.40 & 347.97 & 14.80 & 398.48 & 2.43 \\
\hline & 9 & 408.95 & 364.49 & 10.87 & 374.79 & 8.35 \\
\hline \multirow[t]{2}{*}{3} & 9 & 408.95 & 364.49 & 10.87 & 373.11 & 8.76 \\
\hline & 10 & 431.03 & 397.46 & 7.79 & 397.86 & 7.70 \\
\hline \multicolumn{3}{|c|}{ Mean absolute error } & & 12.48 & & 5.72 \\
\hline \multicolumn{3}{|c|}{ Maximum absolute error } & & 29.75 & & 8.76 \\
\hline
\end{tabular}

Table 5. Identified stiffness results of the 10-storey frame for four different input forces.

\begin{tabular}{ccccccccc}
\hline $\begin{array}{c}\text { Sub- } \\
\text { structure }\end{array}$ & Storey & $\begin{array}{c}\text { Force A } \\
(\mathrm{N})\end{array}$ & $\begin{array}{c}\text { Force B } \\
(\mathrm{N})\end{array}$ & $\begin{array}{c}\text { Force C } \\
(\mathrm{N})\end{array}$ & $\begin{array}{c}\text { Force D } \\
(\mathrm{N})\end{array}$ & $\begin{array}{c}\text { Mean } \\
(\mathrm{N})\end{array}$ & $\begin{array}{c}\text { SD }^{*} \\
(\mathrm{~N})\end{array}$ & $\begin{array}{c}\text { SD/Mean } \\
(\%)\end{array}$ \\
\hline 1 & 1 & 441.33 & 405.99 & 443.52 & 447.15 & 434.50 & 19.16 & 4.41 \\
& 2 & 444.68 & 393.27 & 455.77 & 447.59 & 435.33 & 28.43 & 6.53 \\
& 3 & 420.21 & 389.48 & 429.73 & 426.33 & 416.44 & 18.40 & 4.42 \\
\hline 2 & 3 & 420.66 & 403.95 & 393.11 & 423.03 & 410.19 & 14.20 & 3.46 \\
& 4 & 440.70 & 388.36 & 432.50 & 444.80 & 426.59 & 26.00 & 6.09 \\
& 5 & 393.82 & 371.60 & 417.46 & 395.95 & 394.71 & 18.74 & 4.75 \\
& 6 & 401.10 & 388.45 & 400.04 & 403.27 & 398.21 & 6.65 & 1.67 \\
& 7 & 380.27 & 377.18 & 390.43 & 383.11 & 382.75 & 5.67 & 1.48 \\
& 8 & 396.90 & 401.43 & 396.67 & 398.93 & 398.48 & 2.21 & 0.56 \\
& 9 & 368.63 & 388.70 & 370.47 & 371.38 & 374.79 & 9.34 & 2.49 \\
\hline 3 & 9 & 371.39 & 369.02 & 375.79 & 376.25 & 373.11 & 3.50 & 0.94 \\
& 10 & 399.65 & 396.55 & 394.69 & 400.55 & 397.86 & 2.72 & 0.68 \\
\hline
\end{tabular}

* SD = Standard deviation 
Table 6. Mass values and identification errors of the 10-storey frame based on complete measurements in the experimental study

\begin{tabular}{|c|c|c|c|c|c|}
\hline \multirow[b]{2}{*}{ Storey } & \multirow[b]{2}{*}{$\begin{array}{c}\text { Computed } \\
\text { mass } \\
(\mathrm{kg})\end{array}$} & \multicolumn{2}{|c|}{ CSI } & \multicolumn{2}{|c|}{ Sub-SI } \\
\hline & & $\begin{array}{c}\text { Indentified } \\
\text { mass } \\
(\mathrm{kg})\end{array}$ & $\begin{array}{c}\text { Absolute } \\
\text { error } \\
(\%)\end{array}$ & $\begin{array}{c}\text { Indentified } \\
\text { mass } \\
(\mathrm{kg})\end{array}$ & $\begin{array}{c}\text { Absolute } \\
\text { error } \\
(\%)\end{array}$ \\
\hline 1 & 3.25 & 2.50 & 23.08 & 3.27 & 0.08 \\
\hline 2 & 3.25 & 3.37 & 3.65 & 3.35 & 2.24 \\
\hline 3 & 3.25 & 3.12 & 3.94 & 2.88 & 12.43 \\
\hline 4 & 3.25 & 3.13 & 3.77 & 3.36 & 4.84 \\
\hline 5 & 3.25 & 2.93 & 9.93 & 3.18 & 3.82 \\
\hline 6 & 3.25 & 2.84 & 12.60 & 3.30 & 0.69 \\
\hline 7 & 3.25 & 2.76 & 15.16 & 3.08 & 5.49 \\
\hline 8 & 3.25 & 2.78 & 14.42 & 2.86 & 12.63 \\
\hline 9 & 3.25 & 2.77 & 14.77 & 2.91 & 10.42 \\
\hline 10 & 3.00 & 2.96 & 1.21 & 2.90 & 4.58 \\
\hline \multicolumn{2}{|c|}{ Mean absolute error } & & 10.25 & & 5.72 \\
\hline \multicolumn{2}{|c|}{ Maximum absolute error } & & 23.08 & & 12.63 \\
\hline
\end{tabular}

Table 7. Absolute error (\%) of identified stiffness values of substructure 2 based on incomplete measurements in the experimental study.

\begin{tabular}{|c|c|c|c|}
\hline \multirow{3}{*}{ Floor level } & \multirow{3}{*}{$\begin{array}{c}\text { CSI } \\
\text { Complete measurement } \\
10 \text { sensors }\end{array}$} & \multicolumn{2}{|c|}{ Sub-SI } \\
\hline & & \multicolumn{2}{|c|}{ Incomplete measurement } \\
\hline & & 7 sensors & 4 sensors \\
\hline 3 & 6.58 & 5.16 & 6.91 \\
\hline 4 & 10.59 & 0.81 & 1.92 \\
\hline 5 & 11.65 & 3.23 & 5.62 \\
\hline 6 & 12.22 & 2.26 & 2.59 \\
\hline 7 & 12.31 & 2.46 & 0.90 \\
\hline 8 & 13.30 & 0.78 & 1.91 \\
\hline 9 & 10.97 & 8.25 & 7.83 \\
\hline Mean absolute error & 11.09 & 3.28 & 3.95 \\
\hline Maximum absolute error & 13.30 & 8.25 & 7.83 \\
\hline
\end{tabular}

